\title{
Aproximación a la reforma constitucional de Nicaragua
}

\section{Manuel Gerpe Landín}

Universitat Autònoma de Barcelona. Departament de Ciència Política i de Dret Públic.

Grup d'Estudis Polítics Centramericans. 08193 Bellaterra (Barcelona). Spain

Joan Vintró Castells

Universitat de Barcelona. Departament de Ciència Politica i de Dret Conscitucional.

Barcelona. Spain

\section{Resumen}

El artículo analiza la reforma de la Constitución nicaragüense de 1987 aprobada en 1995. En la parte dogmática se sitúa plenamente el texto dentro de la sradición liberal-democrática y se constitucionaliza un marco de relaciones económicas de economía social de mercado. En la parte orgánica se atenúa el presidencialismo, se refuerza el papel del Parlamento y se atribuye una relevante función de contrapeso al poder judicial.

Palabras clave: Constitución, Nicaragua.

Abstract. Approach to the Nicaraguan constitutional reform

The paper analyses the reform of the Nicaraguan Constitution of 1987 , passed on 1995. On the dogmatic side, the tex is completely set in the liberal democratic tradition and a framework of economic relationships based on social market economy is established by the Constitution. On the organic side, presidentialism is weakened, the role of the Parliament is strengthened and an outstanding function as counterbalance is conferred on the judiciary.

Key words: Constitution, Nicaragua.

\author{
Sumario \\ 1. Introducción 3. Parte orgánica \\ 2. Parte dogmática
}

\section{Introducción}

Desde la derrota del Frente Sandinista en las elecciones de febrero de 1990 y el subsiguiente acceso al poder de Doña Violeta Barrios de Chamorro encabezando la heterogénea coalición de la UNO, las fuerzas políticas nicaragüenses y el Gobierno se han planteado en diversas ocasiones la conveniencia de reformar la Constitución de 1987. La tarea no era fácit, por el equilibrio y la polarización política existente entre los dos bioques y por la dificultad de superar los requisitos que la Constitución establece para su reforma. 
A pesar de las dificultades formales y políticas, la mayoría de la Asamblea Nacional emprende, a partir de la segunda mitad de 1993, la reforma de la Constitución. El camino previsiblemente debía ser tortuoso, pero se consideraba que era el único eficiente para revalorizar el rol político de la Asamblea Nacional dentro del sistema y para adecuar las estructuras estatales a las transformaciones que había sufrido la sociedad nicaragüense desde la aprobación de la Constitución de 1987.

El primer paso se dirige a debilitar la rigidez del procedimiento de reforma establecido en la Constitución de 1987, suprimiendo para la reforma parcial el requisito de que la iniciativa debía ser discutida en dos legislaturas (arts. 192 y 195). La modificación, aprobada por la Asamblea el 23 de febrero de 1994, significa también la exteriorización del conflicto con el Ejecutivo, al negarse la presidenta de la república a promulgar la ley de reforma por considerar que la Asamblea se había extralimitado en sus funciones, argumentando con buen criterio que la reforma del procedimiento de reforma era una reforma total y, por tanto, únicamente podf́a hacerlo la Asamblea Nacional Constituyente.

Aunque el conflicto institucional con el ejecutivo se va acentuando, la Asamblea Nacional mantiene con firmeza el iter parlamentario de la reforma. El 25 de noviembre de 1994 se aprueba en primer debate el texto y en segundo debate, y definitivo, el 1 de febrero de 1995 , se produce la aprobación parlamentaria. El texto es remitido a la presidenta de la república para su promulgación y publicación. Transcurrido el plazo para su promulgación y publicación sin que se llegara a un acuerdo, la Asamblea Nacional decide publicar la Ley de Reforma, que entra a regir el 24 de febrero de 1995.

La reacción del ejecutivo, aunque tiene a su favor buenas razones jurídicas en las diferencias que sostiene sobre el procedimiento utilizado, se justifica mucho mejor si atendemos a que, tanto la presidencia de la República como Antonio Lacayo, ministro de la Presidencia, son los grandes afectados por la reforma constitucional: la presidencia por ver limitados notablemente sus poderes y Antonio Lacayo, al verse implicado en una nueva causa de inelegibilidad que le impide presentarse inmediatamente a la elección de presidente de la República.

La rigidez de las posiciones parece conducir el conflicto a un callejón sin salida en los meses inmediatamente posteriores a la publicación. Todos los intentos de mediación y conciliación ensayados fracasan hasta que, como había sucedido en otras ocasiones, se acude al cardenal Obando y Bravo para que arbitre una solución.

Al amparo de su autoridad arbitral, se configura el Acuerdo Político de 14 de junio de 1995, firmado por el ejecutivo y la Asamblea Nacional. El acuerdo dará salida, o cuanto menos dejará adormecida, la tensión institucional. Haciendo una valoración global del mismo, puede admitirse que legitima la obra del Poder de Reforma: no incluye ninguna modificación a su redacción definitiva, y únicamente matiza el ritmo de implementación de las reformas y establece cautelas para garantizar la participación del ejecutivo en la elaboración de las leyes de desarrollo de las reformas constitucionales. 
Con estos singulares antecedentes, pasamos a continuación a hacer una breve descripción de los principales puntos sustantivos de la reforma constitucional de 1995 (Ley 192), cuya interpretación y aplicación queda pendiente del desarrollo de la Ley Marco (Ley 199), que resuelve el conflicto político entre la Asamblea Nacional y la Presidenta de la República.

\section{Parte dogmática}

\section{A. Reforzamiento de la democracia pluralista y representativa}

No puede sin duda negarse a la Constitución nicaragüense de 1987 el carácter de pluralista y representativa. En este sentido los artículos 5 y 55 contemplaban explícitamente el pluralismo político como principio fundamental y reconocían el derecho a organizar partidos políticos con el fin de optar al poder. Por su parte el artículo 7 definía el Estado como República democrática y representativa. Asimismo, era notoria a lo largo de todo el texto constitucional la ausencia de toda referencia a un partido único o partido guía.

Sin embargo, tampoco puede ignorarse que la Constitución nicaragüense, en su artículo 2, además de señalar que el pueblo ejercía el poder por medio de representantes libremente elegidos por sufragio universal, establecía que el pueblo ejerce también el poder udirectamente» sin más precisiones acerca de los instrumentos de esta participación directa. Por otro lado, la Constitución introducía, de forma un tanto implícita pero perceptible, en el artículo 4 y en otros precepros, un concepto de pueblo que no se identifica con el conjunto de todos los ciudadanos sino con los sectores económicamente más desfavorecidos del país. Si a eso se le añade que el propio artículo 4 afirmaba que el pueblo nicaragüense ha constituido un nuevo Estado para defender sus conquistas revolucionarias y que la regulación constitucional de la defensa reposa sobre la figura del Ejército Popular Sandinista como brazo armado del pueblo y sobre la obligación del Estado de armar al pueblo para defender las conquistas revolucionarias, la singularidad del texto constitucional nicaragüense en este ámbito salta a la vista de inmediato.

Todo ello tiene su explicación histórica en el proceso revolucionario nicaragüense que culminó en 1979 con la caída del dictador Somoza merced al esfuerzo conjunto de los sectores democráticos nicaragüenses, entre los que jugaron un papel decisivo las clases populares y el Frente Sandinista de Liberación Nacional. Este mantenimiento del protagonismo y de la hegemonía política de las clases populares y del Frente Sandinista, en los años posteriores al triunfo revolucionario, cuyas causas y vicisitudes no corresponde analizar en este trabajo, determinaron en la práctica una creciente identificaciónconfusión entre pueblo-Frente Sandinista-Ejército Sandinista-Gobierno-Estado de la que la Constitución de 1987 - a pesar de su reconocimienso del pluralismo, la democracia representativa e incluso del carácter nacional y no partidista del Ejército (art. 95) - no consigue sustraerse plenamente. La conclusión es que en la Constitución se encuentran presentes dos lógicas y tradiciones: la 
tradición liberal-democrática que pone el énfasis en la democracia pluralista y representativa y que tal vez sea la que tenga un reconocimiento formal más explícito; y la tradición política de la izquierda revolucionaria con una concepción un tanto mística de la figura del pueblo y con la incorporación de soluciones institucionales no acordes con los principios del pluralismo polírico y la democracia representativa.

Frente a estas características del texto constitucional de 1987 en la reforma de 1995 destacan una serie de puntos fundamentales que a continuación se reseñan y que, como podrá comprobarse, van en la línea de reforzar la democracia representativa y de eliminar los posibles obstáculos a su plena consolidación:

1. Introducción de dos precisiones importantes en el artículo 2 relativas al ejercicio del poder político. Por una parte, se mantiene que el poder político lo ejerce el pueblo por medio de sus representantes libremente elegidos por sufragio universal pero se añade que «ninguna otra persona o reunión de personas pueda arrogarse este poder o representación". Por otra parte, se concreta la forma mediante la cual el pueblo podrá ejercer de manera directa el poder político, a saber, referéndum y plebiscito de acuerdo con las leyes en la materia. En cuanto al reconocimiento del pluralismo político, puede observarse que en el artículo 5 persiste la previsión de exclusiones. La nueva fórmula propuesta para excluir organizaciones políticas ofrece algo más de seguridad jurídica, ya que no serán admitidas aquellas que upretenden el restablecimiento de todo tipo de dicradura o de cualquier sistema antidemocrático». Desaparece, por lo tanto, la ambigua referencia a las organizaciones "que pretendan el retorno al pasado" o "establecer un sistema político similarn. Con todo, es destacable este mantenimiento de límites ideológicos al pluralismo político cuando en la práctica estas cláusulas son inoperantes o plantean graves problemas jurídicos y políticos en el momento de su aplicación.

2. La supresión de la idea de Estado revolucionario del pueblo presente en el artículo 4 del texto de 1987 y su sustitución por una fórmula relativa al papel del Estado en la promoción del progreso social y económico parangonable con otras Constituciones que recogen los principios del Estado Social de Derecho. Precisamente la definición de Nicaragua como Estado Social de Derecho aparece por primera vez en el nuevo artículo 130 de la Constitución reformada.

3. Una nueva denominación y configuración del Ejército. La reforma operada en el Título 5 de la Constitución introduce diversos cambios significativos en este ámbito. El término "Sandinista" desaparece de la denominación y de este modo la defensa nacional es encomendada al «Ejército de Nicaraguan. En cuanto a la configuración del Ejércico, se enfatiza su carácter "profesional, apolítico, apartidista y no deliberante" y su sumisión al poder civil. Paralelamente, se suprimen todas las referencias del texto de 1987 al pueblo en armas como instrumento de defensa de las conquistas 
revolucionarias y la posible intervención del Ejército en asuntos internos se reserva a supuestos de grave desestabilización interior o de calamidades naturales, es decir, al ámbito de los estados excepcionales configurados en la Ley de Emergencia.

Por úlrimo, no puede dejar de citarse en esta mención de las cuestiones relativas a la defensa la supresión del servicio militar obligatorio (art. 96), uno de los temas estrella de la campaña electoral de 1990.

\section{B. Ampliacion de los derechos de los ciudadanos y de las garantías de su ejercicio}

La relación de derechos y libertades de la Constitución de Nicaragua de 1987 es muy amplia y detallada y está contenida en el Título IV de la misma. Los derechos individuales, sociales, económicos y culturales, presentes en la mayor parte de las Constituciones democráticas contemporáneas, son también reconocidos, sin excepción destacable, por la norma fundamental nicaragüense que otorga particular relieve a los derechos de carácter social entendidos como mandatos dirigidos a los poderes públicos con el objetivo de alcanzar mayores cotas de igualdad y bienestar. Por otra parte, el texto constitucional declara la plena vigencia en Nicaragua de los derechos reconocidos por las declaraciones y los pactos internacionales en materia de derechos humanos (art. 46).

En cuanto a las garantías del ejercicio de tales derechos, puede señalarse, además de la propia trascendencia de su reconocimiento en la norma jurídica superior, las reservas de ley para la regulación concreta de los derechos más importantes y la garantía jurisdiccional del recurso de amparo ante la Corte Suprema de Justicia que puede ser interpuesto por cualquier ciudadano frente a una violación por parte de los poderes públicos de cualquiera de los derechos recogidos en la Constitución (art. 188).

La reforma constitucional de 1995 no pretende ningún recorte al extenso catálogo de derechos y garantías del texto constitucional nicaragüense, sino que más bien trata de ampliarlo. Tomemos algunos ejemplos particularmente reveladores como son los relativos al ámbito del poder judicial, al derecho de propiedad, a la reserva de ley en materia tributaria y a los nuevos instrumentos de protección de los derechos.

En cuanto al primer punto, merecen destacarse tres modificaciones principales que afectan, entre otros, a los artículos 26,34 y 93 :

1. La competencia exclusiva del poder judicial para autorizar la entrada en un domicilio. Con todo, debe strbrayarse que la relación de supuestos en los que no es precisa la autorización judicial para entrar en un domicilio está regulada en unos términos muy amplios y propiciadores de una aplicación abusiva por parte de las fuerzas de seguridad.

2. La supresión de la admisibilidad de las jurisdicciones excepcionales.

3. La prohibición de que los tribunales militares puedan juzgar a civiles, quedando la competencia de la jurisdicción militar limitada a los delitos y fal- 
tas militares cometidos por miembros de las fuerzas armadas en el ejercicio de sus funciones.

Por lo que respecta al derecho de propiedad, las modificaciones en el artícuto 5 y sobre todo en el artículo 44 ponen especial énfasis en situar en un mismo plano de igualdad los diversos tipos de propiedad que la Constitución reconoce (privada, pública, asociativa, cooperativa, comunitaria) y en garantizar el derecho a la propiedad privada de bienes muebles e inmuebles y de los instrumentos y medios de producción, la prohibición de la confiscación de bienes y la garantía de la indemnización justa en los supuestos de expropiación por motivos de la necesaria función social de la propiedad.

El tercer ejemplo se proyecta sobre uno de los elementos de la Constitución. de 1987 que más controversia juridica y política ha desatado a raíz de la interpretación y de la aplicación que tanto el ejecutivo sandinista como el presidido por Violeta Chamorro han hecho de los artículos 115 (creación por ley de los impuestos) y 150.4 (decretos ejecutivos con fuerza de ley por parte del presidente en materia fiscal). Esta interpretación ha provocado que en realidad el artículo 115 haya quedado prácticamente sin efecto y por lo tanto los ciudadanos se han visto desprovistos de la garantía de la reserva de ley parlamentaria en una cuestión tan trascendente para sus intereses como es la de las obligaciones tributarias. A ello pone remedio la reforma del artículo 150.4 al eliminarse la mención a los decretos ejecutivos en materia fiscal.

Por útimo, es preciso subrayar que la reforma constitucional de 1995 introduce un nuevo cauce institucional para la protección y defensa de los derechos de los ciudadanos a través de la creación de la figura del Procurador de los Derechos Humanos (art. 138.9). Con ello se incluye en el ordenamiento nicaragüense una institución similar al ombudsman o defensor del pueblo, tanto en su procedimiento de elección como en sus atribuciones y que ya tiene antecedentes en otros países latinoamericanos. A propósito de las garantías del ejercicio de los derechos constitucionales debe, sin embargo, consignarse que, a pesar del reforzamiento del papel de la Asamblea Nacional en la decisión sobre la declaración del Estado de Emergencia (nuevo artículo 150.9), la reforma no ha afectado a la amplitud de la suspensión de derechos (art. 186), entre los ctrales parece abusivo incluir los referidos al recurso de amparo (art. 45), asociacionismo profesional o corporativo (art. 49) y las condiciones de trabajo (art. 82).

\section{Opción por la economía de mercado y el Estado social}

En algunos preceptos, la Constitución nicaragüense de 1987 parecía querer encontrar en la regulación del sistema económico un equilibrio entre el principio de la dirección y planificación estatal de la economía (art. 99) y cl principio de la economía mixta que garantiza la existencia de diversos tipos de propiedad, y entre ellos la propiedad privada (art. 5 y 103). Ahora bien, la taj̧ante afirmación del artículo 4 según la cual «el Estado es el principal instrumento del pueblo para eliminar toda forma de sumisión y explotación" y la reserva al área 
estatal de manera irrenunciable (arts. 99 y 102) de la banca central, el sistema financiero estatal, los seguros, el comercio exterior y la explotación de los recursos naturales revelan que el texto constitucional nicaragüense, sin caer plenamente en la lógica del constitucionalismo socialista, optaba por otorgar al Estado un papel de dirección y de intervención en la vida económica muy superior a las pautas de lo que comúnmente se conoce como economía de mercado.

La orientación de la reforma constitucional de 1995 va en una línea contraria y viene en cierto modo a formalizar jurídicamente la evolución de la realidad económica nicaragüense en el período posterior a las elecciones de 1990 . Así, entre las previsiones más destacables de la reforma en lo relativo a la Constitución económica deben citarse las siguientes:

1. La eliminación del carácter dirigente del Estado en la economia (arts. 4 y 99), enfatizándose que «el ejercicio de las actividades económicas corresponde primordialmente a los particulares".

2. El respeto y la garantía en un mismo plano de igualdad de los diversos tipos de propiedad (arts. 99 y 104).

3. El reconocimiento de la libertad de empresa y del pleno ejercicio de las actividades económicas (arts. 99 y 104).

4. La admisibilidad clara de la actuación privada en la banca, seguros y comercio exterior (art. 99).

Ahora bien, esta abierta opción por la economfa de mercado no va en detrimento de la inclusión de los elementos más caracteristicos del Estado social. Cabe recordar a este respecto que la definición del Estado como sacialse incorpora en el nuevo artículo 130. En este sentido, y coherentemente con esta definición de Estado social, el texto constitucional resultante tras la reforma mantiene o incorpora, entre otros, los siguientes aspectos:

1. La responsabilidad del Estado de promover el desarrollo integral del país y de garantizar la democracia económica y social (art. 99).

2. El papel del Estado para asegurar el derecho a la educación y a la salud (art. 119,121 y 59).

3. La significación estratégica de la reforma agraria para la transformación económica del país y para dar satisfacción a las reivindicaciones históricas de los campesinos (art. 106 y 107).

4. La intervención del Estado para asegurar la explotación racional de los recursos naturales (art. 102).

\section{Conclusión}

A modo de conclusión puede afirmarse que la reforma constitucional de 1995, en lo relativo a la parte dogmática, pretende eliminar todos los elementos singulares del texto de 1987 más discordantes con la tradición liberal-democrática $y$, al propio tiempo, constitucionalizar un marco de relaciones económicas de economía social de mercado. 


\section{Parte orgánica}

\section{A. Dignificación de la clase politica}

Una de las finalidades de la reforma constitucional es dignificar la vida pública, evitando que la República sea considerada como un bien familiar o patrimonial. Para conseguirlo, se adoptan un conjunto de medidas tendentes a impedir el nepotismo y la corrupción.

Para limitar el nepotismo, práctica habitual y de la que es un buen ejemplo el de Toño Lacayo, yerno de la presidenta de la República, se prohibe expresamente que puedan ser electos presidente de la República ni ministro, viceministro, presidente o director de entes autónomos y gubernamentales y cmbajadores los parientes del presidente de la República dentro del cuarto grado de consanguinidad o segundo de afinidad. Una prohibición que se generaliza para los nombramientos de todos los poderes del Estado y orras instituciones constitucionales, concretándola en que no puede existir tal grado de parentesco con la autoridad que hace el nombramiento y, en su caso, con la persona de donde hubiera emanado esta autoridad (arts. 130 y 152).

Frente a la corrupción, una de las medidas que se adoptan es la de establecer que ninguna de las autoridades públicas, en su sentido más amplio, pueda obtener concesión alguna del Estado. Tampoco pueden ser apoderados o gestores de empresas públicas o privadas, nacionales o extranjeras, en contrataciones de éstas con el Estado. La violación de esta disposición anula las concesiones o ventajas obtenidas y causa la pérdida de la representación y el cargo (art. 130).

A ello debe añadirse un riguroso tratamiento de las causas de inclegibilidad que, aunque globalmente responda a purificar la representación, no esconde un cierto ánimo de depuración política (arts. 134 y 147). Ejemplos de tales medidas son las limitaciones establecidas para los que hubieran renunciado anteriormente a la nacionalidad nicaragüense o no hubieran residido o trabajado en forma continua en el pás los cinco años anteriores a la elección.

\section{B. Cambios en la normativa electoral}

Aunque la valoración de los sistemas clectorales corresponde a otras comunicaciones, cabe dejar constancia de una obviedad de doble fllo: se actúa sobre una realidad en la que la presidenta accede al cargo con unos apoyos electorales que casi inmediatamente abandona en su tarea de gobierno, de manera que una buena parte de los mismos constituyen el núcleo duro de la oposición a su gestión, y con una Asamblea Nacional con un pluralismo exacerbado que, en muchos momentos, impide incluso su regular constitución.

Lo primero se puede considerar que favorece modificaciones en favor de flexibilizar, dando mayor margen a la autonomía partidista, la presentación de candidaturas a la Presidencia y abrir un margen posterior para las grandes alianzas. Lo segundo, lo que afecta a la atomización de la representación en la Asamblea, deberá corregirse reforzando la tendencia mayoritaria del sistema proporcional, que no se discute. 
Desde tal lectura se justifica la coherencia de la reforma en favor de la elección popular directa del presidente en un sistema de doble vuelta. En la primera para resultar electo se requiere obtener al menos el cuarenta y cinco por ciento de los votos válidos. De no darse, a la segunda vuelta accederían únicamente los dos candidatos más votados en la primera, resultando elegido el que mayor número de votos obtuviera. La papeleta de presidente contiene la de vicepresidente, una inclusión que gana peso en la medida que en el conjunto del proyecto se refuerzan las funciones de gobierno del mismo (art. 147).

Con la finalidad de corregir la atomización de la representación, las medidas que se proponen en relación con la elección de los miembros de la Asamblea Nacional son: se mantiene el número de 90 diputados, de los cuales 20 serán elegidos en circunscripción electoral nacional y los 70 restantes en los departamentos (antes la circunscripción era más amplia, la región) y regiones autónomas (art. 132). Disminuyendo el tamaño electoral de la circunscripción parece que deberá también potenciarse el efecto mayoritario de la fórmula electoral.

A élo debe añadirse que también establece la limitación temporal del mandato del presidente y de los representantes, que pasarían de 6 a 5 años (arts. 148 y 136).

\section{Atenuación del presidencialismo}

Para atenuar el presidencialismo, intensísimo en la Constitución de 1987 y con ribetes dudosamente coherentes con el presidencialismo democrático, se propone una doble vía de corrección: la más explícita, potenciar la función decisoria de la Asamblea Nacional; y la menos explícita, potenciar la función ejecutiva del Consejo de Ministros.

10 El debilitamiento de las funciones del presidente de la República tiene como materias significativas las siguientes:

a) Disminución de sus facultades electivas: al margen de los rigurosos requisitos de elegibilidad a los que hemos hecho referencia y que aquí afectaría a los miembros del Gobierno y altos cargos de la Administración, interesa destacar que se debilita la potestad del presidente en lo que afecra a los miembros del Consejo Supremo elecroral, al procurador de los Derechos Humanos, al contralor general de la República, y al procurador general de Justicia. En todos estos últimos supuestos el presidente mantiene el derecho de presentar lista de candidatos pero en competencia con listas presentadas por los diputados de la Asamblea Nacional, siendo esta última la que termina decidiendo por votación.

- b) Una muy intensa pérdida de las facultades normativas, especialmente de dar normas con rango de ley, que se trasladan a la Asamblea Nacional. Las principales expresiones de este fenómeno son: 
1. Aunque se mantiene la potestad de veto parcial o total de las leyes, se introducen dos matizaciones: la primera establece unas normas que no pueden ser vetadas, en cuanto están excluidas de la sanción por el presidente, incluyéndose dentro de ellas las reformas a la Constitución, las leyes constitucionales y los decretos aprobados por la Asamblea Nacional (Art. 141); la segunda intenta evitar que el presidente pueda paralizar con su inactividad la entrada en vigor de las leyes y se concreta en que el presidente de la Asamblea, transcurrido el plazo que la Constitución da ał presidente de la República para publicar, mandará publicar la norma por cualquier medio de comunicación social cscrito, entrando en vigencia desde dicha fecha (arts. 141 y 142).

2. Suprime la atribución del presidente de dictar decretos ejecutivos con fuerza de ley en materias de carácter fiscal, quedando limitada su potestad a lo administrativo. Aquí se encontraba una de las principales quiebras del principio de legalidad que permitía la Constitución de 1987.

3. Suprime la posibilidad de delegar las facultades legislativas al presidente de la República en fases de receso de la Asamblea Nacional.

4. Acota las facultades del presidente en relación con tratados, convenios y acuerdos internacionales al someterlos a la preceptiva deliberación y aprobación por la Asamblea Nacional (arts. 138.12 y 150.8).

5. Intensa limiración de la potestad presidencial de reglamentar las leyes. Se opta por una concepción muy tígida del principio de vinculación positiva a la Lcy, que se traduce en que la potestad se ejercerá cuando la ley expresamente lo determine (art. 141). A ello se añade que, en tales supuestos, la reglamentación debe haceria el presidente en un plazo no mayor de sesenta días (art. 150.9), y que de no hacerlo será la propia Asamblea Nacional la que ejercerá la potestad reglamentaria (art. 141).

6. Somete a reserva de Ley, lo cual tiene efectos destacados sobre la capacidad organizacional y funcional del presidente, cl número de ministerios, la organización de los mismos y las competencias de los ministerios. La reserva de Ley se extiende a los entes autónomos y gubernamentales, a los bancos estatales y demás instituciones financieras del Estado (art. 151).

7. Refuerzo de las funciones presupuestarias de la Asamblea Nacional. Según los nuevos artículos 138.6 y 150.5 corresponde al presidente de la República elaborar el proyecto de ley de presupuestos y a la Asamblea Nacional aprobarlo. Con ello se enfatiza que la determinación del contenido concreto y definitivo del presupuesto corresponde a la Asamblca y se pretende evitar una práctica de años anteriores en la que el poder legislativo se limitaba a conocer y a aprobar formalmente el presupuesto. En el mismo sentido debe interpretarse la desaparición en el artículo I 85 de la facultad presidencial de aprobar el presupuesto durante la vigencia del Estado de Emergencia. 
Aún limitándonos a las expresiones anteriores, pocas dudas caben que el fenómeno de desapoderamiento del presidente en favor de la Asamblea Nacional es muy intenso: la Asamblea adquiere el monopolio de la función legislativa, se potencian sus facultades en materia presupuestaria y se amplía notablemente la reserva de ley.

c) Un tercer aspecto altamente significativo para Nicaragua es que se limita la libre disposición del presidente sobre los estados excepcionales, sobre la emergencia constitucional. Se mantiene la exclusiva iniciativa del presidente pero el Decreto debe ser sometido en el plazo de 72 horas a la Cámara que puede ratificarlo, modificarlo o rechazarlo (art. 150.9).

$2^{\circ}$ La vertiente menos explícita de la atenuación del presidencialismo es la constitucionalización del Consejo de Ministros. Paradójicamente, aunque sea la menos explícita es la que potencialmente encierra mayor contenido reformador.

Es por ello que el tema debe ser tratado con mucha cautela para evitar conclusiones precipitadas en favor de fórmulas semipresidenciales o extremadamente limitativas de la función de dirección política del presidente de la República.

El primer dato a considerar es que durante el mandato de Dońa Violeta se ha intensificado progresivamente la práctica de que el ministro de Presidencia, Antonio Lacayo, ejerce una función similar a la de un primer ministro, especialmente en política interna, y que también se han normalizado las reuniones del Consejo de Ministros. El talante personal de Doña Violeta parece conjugar bien con el refuerzo de su imagen interna de poder super-partes.

La Ley de reforma ha constitucionalizado tal práctica, pero la configura de forma notablemente distinta a como hasta ahora actúa. El cambio más notable son las limitaciones que establece a la discrecionalidad del presidente de la República. Ello se acredita en dos datos: el primero, regulación constitucional y remisión a ley de su regulación y funciones; el segundo, de gran trascendencia, afecta a la Presidencia del Consejo, ésta corresponde al presidente de la República, y en su defecto al vicepresidente de la República, que es miembro de derecho del Consejo junto a los ministros de Estado (art. 151).

El segundo dato es la potenciación de los mecanismos de control parlamentario sobre el Consejo de Ministros y los ministros. Aunque no requieren de la confranza de la Asamblea Nacional, se introducen controles por vía de comisiones de investigación y de interpelación parlamentaria que tienen la vocación de que sean vinculantes para el presidente de la República (art. 138.4 y 151). Por último, debe destacarse la incorporación del refrendo de actos del presidente de la República: sus decretos y providencias deben ser refrendados por los ministros de Estado de las respectivas ramas, salvo aquellos acuerdos que se refieran a nombramientos o remoción de sus ministros o viceministros de Estado (art. 151). 


\section{Reforzamiento de los órganos jurisdiccionales}

1. Uno de los rasgos más relevantes de las democracias contemporáneas es la potenciación de la función jurisdiccional. A ella no cumple únicamente la solución de los conflictos entre particulares y entre los particulares y la Administración, sino que también se ha traducido en órganos que controlan al legislador y al ejecutivo y que resuelven también conflictos que puedan darse entre ellos. Pacíficamente podríamos admitir que los controles jurisdiccionales son complementarios de los tradicionalmente denominados políticos o interinstitucionales, y que no constituiría una osadía, incluso en los sistemas parlamentarios, el afirmar la preeminencia del control jurisdiccional sobre el control político.

Podemos admitir que esta lógica es la seguida por la reforma constitucional. Y, coherentemente, se refuerza la independencia de los organos jurisdiccionales, se extiende la reserva de jurisdicción y se sientan las bases para una nueva organización del poder judicial.

2. Los tribunales de justicia se conciben como un sistema unitario cuyo órgano superior es la Corte Suprema de Justicia.

Las garantías esenciales de su autonomía son fijar por la Constitución que el poder judicial recibirá anualmente no menos del $4 \%$ del presupuesto general de la República y el establecimiento de la carrera judiciał (art. 159).

La independencia de los jueces se intenta reforzar estableciendo un rígido sistema de incompatibilidades y de causas de inelegibilidad (art. 161), y disminuyendo, casi anulando, la incidencia del Ejecutivo en el nombramiento de los miembros del poder judicial, que queda limitado a proponer a la Asamblea Nacional listas de candidatos para la elección de los magistrados de la Corte Suprema en competencia con listas que pueden presentar los diputados, siendo la Asamblea Nacional la que decide.

3. La reserva de jurisdicción se extiende mediante la creación de la sala de lo contencioso-administrativo en la Corte Suprema (art. 163). La creación de tal sala debe ser interpretada como un primer paso para que posteriormente la ley desarrolle la jurisdicción contencioso-administrativa. Una creación que consideramos deberá ir acompañada, como se defendía en alguna propuesta de reforma, de la creación de una especifica jurisdicción laboral o social.

A ello debe añadirse que la jurisdicción militar, aunque se mantiene, sólo conocerá las faltas y los delitos estrictamente militares, sin perjuicio de las instancias y recursos ante la Corte Suprema de Justicia (art. 159).

4. Aunque existieron iniciativas para crear una jurisdicción constitucional de nuevo cuño, concentrada, finalmente se ha optado por mantener $\mathrm{cl} \mathrm{mismo}$ modelo, reformar su organización y ampliar sus competencias.

En relación con la organización se crea una sala de lo constitucional en la Corte Suprema y se atribuyen al pleno el conocimiento y la resolución de 
los recursos de inconstitucionalidad de la Ley, y los conflictos de competencia y constitucionalidad entre los poderes del Estado (art. 163), que, en su enumeración pot el art. 164, permite constatar que se incorporan tanto los conflictos de atribuciones entre órganos políticos y administrativos como los de competencia entre órganos centrales y órganos de las regiones autónomas y de los municipios.

5. Aunque no sean órganos jurisdiccionales, la voluntad de garantizar el principio de legalidad y los derechos fundamentales y las libertades públicas se evidencia en el refuerzo o la creación de instituciones de garantía a las que se dota de independencia en el ejercicio de su función (Procuraduría General de Justicia, Procuraduria de Derechos Humanos y Contraloría General de la República).

\section{E. Conclusion}

Aun admitiendo que los proponentes de la reforma conocen el gran debate doctrinal sobre la incidencia de las formas de gobierno en los procesos de transición a la democracia en Latinoamérica - y que tal debate en cuanto toma como núcleo mínimo la necesidad de atenuar el presidencialismo es especialmente eficaz para su proceso de transición, ya que se produce sobre una estructura jurídico-institucional de presidencialismo extremo que ha mostrado su inopcrancia para garantizar la estabilidad - existen buenas razones para concluir que los que proponen las reformas no tienen una concepción sistemática sobre la forma de Gobierno.

Se puede sostener que son causas eminentemente "locales" las que de forma inmediata motivan las propuestas de reforma institucional. Al caracterizar las causas como "locales" ponemos en evidencia que son respuestas singulares a problemas concretos que la práctica constitucional ha planteado durante los años de mandato de Doña Violeta y que tales posiciones responden políticamente a la visión que de tales procesos tienen las fuerzas políticas impulsoras de la reforma constitucional.

Sin embargo, es de todos conocido que la forma de gobierno de un sistema político no depende únicamente, ni prioritariamente, de la determinación jurídica de las instituciones y de sus relaciones. No puede desconocerse que la racionalización jurídica tiene un efecto condicionador u orientador de la práctica institucional, pero no la fija o la prederermina totalmente. En último término deberán ser los actores políticos y sus prácticas los que doten de coherencia sistemática al entramado institucional. Son ellos, actuando institucionalmente, los que determinarán la forma de gobierno.

Desde taies presupuestos, nuestras conclusiones serían:

1. Las reformas propuestas atenúan los poderes del presidente y refuerzan los de la Asamblea Nacional y los del Consejo de Ministros. Es pues notoria la 
voluntad de reformular un nuevo equilibrio de poderes que mitigue el presidencialismo.

2. Las reformas no rompen la esencia de un sistema presidencial: legitimación popular directa del presidente y dependencia del Consejo de Ministros del presidente de la República. El Gobierno no precisa de la confianza de ia Asamblea ni es responsable políticamente ante ella.

3. Se ofrecen datos normativos suficientes para considerar que por vía convencional el sistema pueda integrar tendencias parlamentarias: lo que requeriría potenciar la autonomía del Consejo de Ministros en relación con el presidente de la República y aumentar su relación fiduciaria con cl Parlamento. Una tendencia que tendría menos dificultades de no adjudicarse al vicepresidente de la República la Presidencia del Consejo de Ministros.

4. Es muy relevante la función de contrapeso que se atribuye al poder judicial, que, como sucede en las democracias pluralistas estables, pasa a ser el principal controlador de la actuación de los poderes públicos y et árbitro de sus conflictos de atribuciones o de competencias. 